\title{
Evaluation of Helicobacter pylori eradication on the course of childhood nephrotic syndrome and its response to treatment
}

\author{
Hassan Bazmamoun ${ }^{1}$, Danyal Isapour ${ }^{2}$, Zahra Sanaei ${ }^{3}$, Rahimpour Amiri ${ }^{4 *(\mathbb{D}}$ \\ Received: 19 Jun 2020 \\ Published: 21 Apr 2021
}

\section{Abstract}

Background: Idiopathic nephrotic syndrome is one of the most common glomerular diseases, which may be secondary to infections or systemic diseases. The aim of this study was to evaluate the effect of Helicobacter pylori (H. pylori) eradication on childhood nephrotic syndrome.

Methods: In this randomized controlled clinical trial study, 38 children with concomitant idiopathic nephrotic syndrome and $H$. pylori infection were divided into 2 equal groups; the intervention group received a cotreatment for both diseases and the control group received only nephrotic syndrome treatment. Patients were followed for 6 months. Data were analyzed using SPSS 21 software. Chi square test, Fisher exact test, and student $\mathrm{t}$ test were used. $\mathrm{P}$ value $<0.05$ was considered statistically significant.

Results: The mean interval time from treatment to the recovery of nephrotic syndrome was $48.36 \pm 14.48$ days in the intervention group and 51.68 17.32 days in control groups, which was shorter in the intervention group, but not statistically significant. The recurrence of nephrotic syndrome and the mean number of recurrences in the intervention group were lower than the control group, but were not statistically significant. The frequency of diarrhea in the intervention group was significantly higher than the control group $(\mathrm{p}=0.003)$.

Conclusion: In children with concomitant idiopathic nephrotic syndrome and $H$. pylori infection, the treatment of both diseases may accelerate the recovery and decrease the recurrence of nephrotic syndrome.

Keywords: Nephrotic Syndrome, Helicobacter pylori Infection, Children

Conflicts of Interest: None declared

Funding: This article was supported by the vice chancellor for research and technology of Hamadan University of Medical Sciences (no: 9807185264).

\section{*This work has been published under CC BY-NC-SA 1.0 license. \\ Copyright $₫$ Iran University of Medical Sciences}

Cite this article as: Bazmamoun H, Isapour D, Sanaei Z, Amiri R. Evaluation of Helicobacter pylori eradication on the course of childhood nephrotic syndrome and its response to treatment. Med J Islam Repub Iran. 2021 (21 Apr);35:52. https://doi.org/10.47176/mjiri.35.52

\section{Introduction}

Nephrotic syndrome is the most common manifestation of glomerular disease in children. Its prevalence varies in different communities, ranging from 1.15 to 16.9 cases per 100000 populations. Its diagnosis is often clinical and is based on the criteria of hypoalbuminemia, hyperlipidemia, generalized edema, and proteinuria in the nephrotic range. Nephrotic syndrome has congenital and acquired types. Acquired types can be primary (idiopathic) or secondary to systemic diseases, genetic disorders, and infections (1,

Corresponding author:Dr Rahimpour Amiri, r.amiri@umsha.ac.ir

1. Department of Pediatric Gastroenterology, Hamadan University of Medical Sciences, Hamadan, Iran

2. Department of Pediatrics, Hamadan University of Medical Sciences, Hamadan, Iran

3. Department of Community Medicine, Education Development Office, Hamadan University of Medical Sciences, Hamadan, Iran

4. Department of Pediatric Nephrology, Hamadan University of Medical Sciences, Hamadan, Iran
2).

The primary form of the disease is responsible for most childhood nephrotic syndrome (3) and the therapeutic response to steroids determines its prognosis. The treatment of the disease is performed with high doses of corticosteroids, which may have unintended side effects $(1,3)$.

Helicobacter pylori (H. pylori) is more common in developing countries, affecting about half of the world's population. This bacterium infects the patient early in life,

$\uparrow$ What is "already known" in this topic:

Some studies suggested that helicobacter pylori infection plays a role in the development of nephropathy.

$\rightarrow$ What this article adds:

In children with concomitant idiopathic nephrotic syndrome and Helicobacter pylori infection, the treatment of both diseases may accelerate the recovery and decrease the recurrence of nephrotic syndrome. 
and without treatment it often remains in the body for the rest of life (4).

Evidences that suggest bacteria play a role in the development of nephropathy is increasing (5-7). Some studies indicate that $H$. pylori infection by influencing the production and glycosylation of IgA1 aggravates renal function and makes more severe degrees of antigen deposition in IgA nephropathy $(8,9,10)$.

Regarding the high prevalence of $H$. pylori infection and its possible role in nephrotic syndrome, this study was conducted to evaluate the effect of $H$. pylori eradication on childhood nephrotic syndrome.

\section{Methods}

In this randomized clinical trial study, all children aged 2-14 years with idiopathic nephrotic syndrome (after confirmation of the diagnosis by a pediatric nephrologist) who referred to the Pediatric Nephrology Clinic of Besat hospital in Hamadan, Iran, from 2017 to 2019, were evaluated by fecal antigen test for $H$. pylori infection using Elisa method and Generic assays kit.

In positive cases, upper endoscopy and gastric biopsy with Giemsa staining were done and if the diagnosis of $H$. pylori infection was confirmed, they entered the study after obtaining informed consent from all patients or their parents. The criteria for the diagnosis of nephrotic syndrome were serum albumin less than $2.5 \mathrm{~g} / \mathrm{dL}$, hyperlipidemia, generalized edema, and proteinuria in the nephrotic range, including protein levels greater than $40 \mathrm{mg} /$ $\mathrm{m}^{2} / \mathrm{h}$ in 24-hour urine or protein-to-creatinine ratio more than 2 to 3 in the morning random urine sample.

Inclusion criteria included all 2-14-year-old children who had been diagnosed with idiopathic nephrotic syndrome by a pediatric nephrologist and had been confirmed to have H. pylori infection with upper endoscopy and gastric biopsy.

Exclusion criteria were as follows: (1) children with the diagnosis of congenital nephrotic syndrome; (2) secondary nephrotic syndrome to immune disorders, infectious diseases, medications, and other toxins; (3) association of glomerulonephritis, renal failure, and hypertension with nephrotic syndrome; (4) patients who have been taking antibiotics or antiacid medications for the past month; (5) patients who were unable to continue treatment due to drug complications; and (6) patients who did not cooperate.

Patients' demographic data were collected using a checklist.

In this study, all patients were included in the study by census during the study period and the sample size was not determined due to the small number of cases. Then, patients were randomly divided into 2 equal groups of intervention and control using a quadruple block. The intervention group received treatment for nephrotic syndrome (prednisone $60 \mathrm{mg} / \mathrm{m}^{2}$ daily) and H. pylori infection (Omeprazole $1 \mathrm{mg} / \mathrm{kg} /$ day, metronidazole $20 \mathrm{mg} /$ $\mathrm{kg} /$ day, amoxicillin $50 \mathrm{mg} / \mathrm{kg} /$ day and bismuth subcitrate $480 \mathrm{mg} / 1.73 \mathrm{~m}^{2}$ ) and the control group received only nephrotic syndrome treatment (prednisone $60 \mathrm{mg} /$ $\mathrm{m}^{2}$ daily). Then, the 2 groups were followed for response to treatment, relapse rate, and drug side effects for 6 months.

Response to treatment for nephrotic syndrome was considered as no protein or trace protein in 3 consecutive urine samples and recurrence of nephrotic syndrome was considered as the reappearance of proteinuria after response to treatment.

Resistance to treatment was also defined as the continuation of proteinuria after a 1-month treatment (11).

Eradication of $H$. pylori was considered as negative $H$. pylori antigen in stool 1 month after the treatment of $H$. pylori infection (12).

This study was conducted with the approval of the Ethics Committee of Hamadan University of Medical Sciences, IR.UMSHA.REC.1397.959, and IRCT201202150090 $14 \mathrm{~N} 268$.

In this study, quantitative variables were presented by mean and standard deviation; and qualitative variables were described by percent. Frequency of sex and recurrence of the disease were analyzed by chi square test. Student $\mathrm{t}$ test was used to compare the mean age, urine protein/ creatinine ratio, and recovery time. The frequency of recurrence was assessed by Fisher exact test. Data were analyzed using SPSS 21 software. P value $<.05$ was considered statistically significant.

\section{Results}

A total of 38 children aged 2-14 years with idiopathic nephrotic syndrome equal in the 2 groups were enrolled (Fig. 1).

Patients in both groups were similar in terms of age, sex, and urine protein / creatinine ratio, and there was no statistically significant difference between the 2 groups (Table 1).

A total of 7 out of 19 patients in the intervention group $(36.8 \%)$ and 12 out of 19 patients in the control group $(63.2 \%)$ had recurrence of nephrotic syndrome $(p=0.105)$. The mean number of recurrences was $1.143 \pm 0.377$ in the intervention and $1.583 \pm 0.793$ times in the control groups.

In the other word, the number and mean number of recurrences_were lower in the intervention group than in the control group, but the difference between the 2 groups was not statistically significant $(\mathrm{p}=0.197)$; however, the observed power of this test was low (0.253).

The average time from treatment initiation until recovery of nephrotic syndrome in the intervention and control groups was $48.36 \pm 14.48$ and $51.68 \pm 17.32$ days, respectively $(\mathrm{p}=0.526)$, meaning that nephrotic syndrome got better earlier in children in the intervention group, but there was no statistically significant difference between the 2 groups (Table 2).

In terms of drug side effects, diarrhea was observed in 7 patients $(37 \%)$ of the intervention group, but not in the control group, which was not statistically significant $(\mathrm{p}=.003)$.

Abdominal pain was seen in 3 patients $(15.8 \%)$ in the intervention group and in 4 patients $(21.1 \%)$ in the control group, which was not statistically significant $(p=0.670)$. The severity of drug complications was not severe enough in any patient to discontinue treatment. 


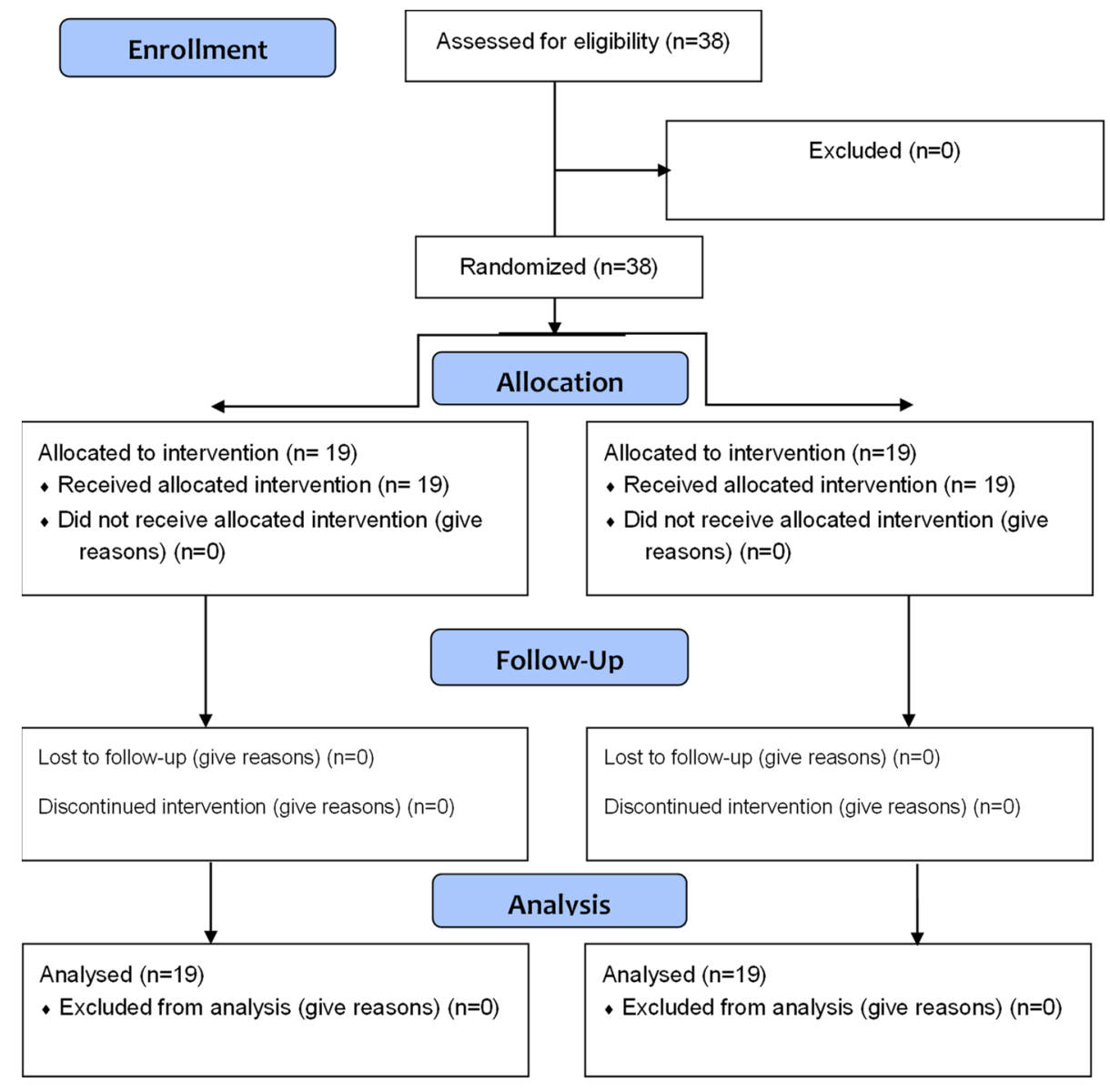

\section{Fig. 1. CONSORT 2010 flow diagram}

Table 1. Distribution of Basic Information of the 2 Groups

\begin{tabular}{lccc}
\hline Characteristics & Intervention $(\mathrm{n}=19)$ & Control $(\mathrm{n}=19)$ & $\mathrm{p}$ \\
\hline Gender, $\mathrm{n}(\%)$ & & & \\
Female & $12(63.2)$ & $10(52.6)$ & 0.511 \\
Male & $7(36.8)$ & $9(47.4)$ & \\
Age (y), mean \pm SD & $5.18 \pm 3.15$ & $6.60 \pm 3.85$ & 0.232 \\
Urine protein/ urine creatinine ratio, mean \pm SD & $2.27 \pm 0.17$ & $2.36 \pm 0.19$ & 0.127 \\
\hline
\end{tabular}

\begin{tabular}{|c|c|c|c|c|}
\hline Characteristics & Intervention $(\mathrm{n}=19)$ & Control $(\mathrm{n}=19)$ & $\bar{p}$ & Observed Power \\
\hline \multicolumn{5}{|l|}{ Relaps, n (\%) } \\
\hline Yes & $7(36.8)$ & $12(63.2)$ & $0.105^{*}$ & \\
\hline No & $12(63.2)$ & $7(36.8)$ & & \\
\hline \multicolumn{5}{|l|}{ Relaps, n (\%) } \\
\hline One occasion & $6(85.7)$ & $7(58.3)$ & & \\
\hline Two occasions & $1(17.3)$ & $3(25.0)$ & $0.333 * *$ & \\
\hline Three occasions & $0(0.0)$ & $2(16.7)$ & & \\
\hline More & $0(0.0)$ & $0(0.0)$ & & \\
\hline Average relaps rate $($ mean $\pm \mathrm{SD})$ & $1.143 \pm 0.377$ & $1.583 \pm 0.793$ & $0.197 * * *$ & 0.253 \\
\hline Period time from treatment to recovery (Day), $(\operatorname{mean} \pm \mathrm{Sd})$ & $48.36 \pm 14.48$ & $51.68 \pm 17.32$ & $0.526 * * * *$ & 0.096 \\
\hline
\end{tabular}

${ }^{*}$ Chi-square test. $\quad * *$ Fisher exact test (Two, three, and more relapses were combined). $\quad * * *$ Mann-Whitney test. ****Student t test.

\section{Discussion}

In this study, the recurrence of nephrotic syndrome and the mean number of recurrences in the intervention group were lower than in the control group, but the difference between the 2 groups was not statistically significant. Also, the average time from treatment initiation until recovery of nephrotic syndrome in the intervention group was shorter than the control group, but this difference was not statistically significant. Regarding drug side effects, the frequency of diarrhea in the intervention group was significantly higher than the control group, but the severity of drug complications was not severe enough in any patient to discontinue treatment.

In the study of Caliskan et al in 2014, the effect of $H$. pylori eradication on proteinuria in adult patients with membranous glomerulonephritis was studied, and it was 
shown that $H$. pylori treatment may decrease proteinuria in patients with membranous nephropathy and also no significant drug side effect was observed during the eradication therapy (5).

In the study of Fahed Ben et al in 2018, the effect of $H$. pylori eradication on proteinuria in children with nephrotic syndrome was studied and it was shown that the eradication of $H$. pylori infection reduced proteinuria (13).

In a cross-sectional study by Mahmoud et al in 2019, children with nephrotic syndrome who received high doses of steroids were more likely to be infected with $H$. $p y$ lori (14).

The study by Zhu et al in 2016 on patients with IgA nephropathy also showed the possible role of $H$. pylori infection by exacerbating mucosal immune response and renal tubular injury in these patients (8).

On the other hand, in the study by Dede et al in 2015, the effect of $H$. pylori eradication on proteinuria in adult patients with primary glomerulonephritis was investigated. There was no significant difference between proteinuria before and after the treatment of $H$. pylori infection, also $H$. pylori eradication did not have a significant effect on the remission of the disease (15).

\section{Conclusion}

In children with concomitant idiopathic nephrotic syndrome and $H$. pylori infection, treatment of both diseases may accelerate the recovery and decrease the recurrence of nephrotic syndrome.

\section{Acknowledgement}

The authors would like to express thanks to all participants for their cooperation and patience in completion of this work.

\section{Conflict of Interests}

The authors declare that they have no competing interests.
8. Zhu TT, Wang L, Wang HL, He Y, Ma X, Fan JM. Helicobacte pylori participates in the pathogenesis of IgA nephropathy. Renal Fail. 2016;38(9):1398-404

9. Yang M, Li FG, Xie XS, Wang SQ, Fan JM. CagA, A major virulence factor of Helicobacter pylori, promotes the production and underglycosylation of IgA1 in DAKIKI cells. Biochem Biophys Res Commun. 2014;444(2):276-81.

10. Li $\mathrm{Q}$, Lin $\mathrm{X}, \mathrm{Wu} \mathrm{Z}, \mathrm{He} \mathrm{L}$, Wang $\mathrm{W}$, Cao $\mathrm{Q}$, et al. Immunohistochemistry analysis of Helicobacter pylori antigen in renal biopsy specimens from patients with glomerulonephritis. Saudi J Kidney Dis Transpl. 2013;24(4):751.

11. Hammad A, Yahia S, Gouida MS, Bakr A, EL-Farahaty RM. Low expression of glucocorticoid receptors in children with steroid-resistant nephrotic syndrome. Pediatr Nephrol. 2013;28(5);759-63

12. Mayo Clinic [Internet]. Mayo Foundation for Medical Education and Research; c1998-2019. Helicobacter pylori (H. pylori) infection: Symptoms and Causes; 2017 May 17 [cited 2019 Jun 27]; [about 3 screens]. Available from: https://www.mayoclinic.org/diseasesconditions/h-pylori/symptoms-causes/syc-20356171

13. Abed FB, Bofarraj M, Khaial FS. The Effect of H. pylori Eradication on Proteinuria in Children with Idiopathic Nephrotic Syndrome at Albida Hospital, Libya. Egypt Pediatr. 2018;35(1\&2):73- 81.

14. Anwar A, Bakr A, EL-SAID A, Wahba Y. Prevalence of helicobacter pylori infection among Egyptian children with nephrotic syndrome: a single center study. Mendeley Data, V2, (2019), doi: 10.17632/3jtgk4zvtg.2. Available from: http://dx.doi.org/10.17632/ 3jtgk4zvtg.2

15. Dede F, Ayli D, Gonul I, Yuksel O, Ozturk R, Yildiz A, et al. The effect of Helicobacter pylori eradication on proteinuria in patients with primary glomerulonephritis. AMS. 2015;11(4):764.

\section{References}

1. Noon DG, Iijima K, Parekh R. Idiopathic Nephrotic syndrome in children. Lancet. 2018;392(10141):61-74.

2. Kikunaga K, Ishikura K, Terano C, Sato M, Komaki F, Hamasaki Y, et al. High incidence of idiopathic nephrotic syndrome in East Asian children: a nationwide survey in Japan (JP-SHINE study). Clin Exp Nephrol. 2017;21(4):651-7.

3. Hussain N, Zello JA, Vasilevska-Ritovska J, Banh TM, Patel VP, Patel $\mathrm{P}$, et al. The rationale and design of insight into nephrotic syndrome: investigating genes, health and therapeutics (INSIGHT): a prospective cohort study of childhood nephrotic syndrome. BMC Nephrol. 2013;14:25.

4. Testerman TL, Morris J. Beyond the stomach: An updated view of Helicobacter pylori pathogenesis, diagnosis, and treatment. World $\mathrm{J}$ Gastroenterol. 2014; 20(36): 12781-12808.

5. Caliskan B, Yazici H, Caliskan Y, Ozluk Y, Gulluoglu M, Kilicaslan I, et al. The effects of Helicobacter pylori eradication on proteinuria in patients with primary glomerulonephritis. Int $\mathrm{J}$ Nephrol. 2014;2014:180690.

6. Kusano K, Inokuchi A, Fujimoto K, et al. Coccoid Helicobacter pylori exists in the palatine tonsils of patients with IgA nephropathy. J Gastroenterol. 2010;45(4):406-12.

7. Nagah Balat M, Fahmy Zanaty MA, EL-Antouny NG, Kamal Ahmed $\mathrm{H}$. Association between proteinuria and active helicobacter pylori infection in non-diabetic patients. Zagazig Univ Med J. January 2019;25(1):79-84 